\title{
Health status, hospitalizations, day procedures, and physician costs associated with body mass index (BMI) levels in Ontario, Canada
}

This article was published in the following Dove Press journal:

ClinicoEconomics and Outcomes Research

23 January 2012

Number of times this article has been viewed

\author{
Jean-Eric Tarride ${ }^{1,2}$ \\ Mahbubul Haq' \\ Valerie H Taylor ${ }^{3}$ \\ Arya M Sharma ${ }^{4}$ \\ Hamid Reza Nakhai-Pour' \\ Daria O'Reilly,2 \\ Feng $\mathrm{Xie}^{1,2}$ \\ Lisa Dolovich ${ }^{2,5,6}$ \\ Ron Goeree ${ }^{1,2}$ \\ 'Programs for Assessment of \\ Technology in Health (PATH) \\ Research Institute, St Joseph's \\ Healthcare Hamilton, Hamilton, \\ Ontario, Canada; ${ }^{2}$ Department \\ of Clinical Epidemiology and \\ Biostatistics, McMaster University, \\ Hamilton, Ontario, Canada; \\ ${ }^{3}$ Department of Psychiatry, University \\ of Toronto, Ontario, Canada; \\ ${ }^{4}$ Department of Medicine, University \\ of Alberta, Edmonton, Alberta, \\ Canada; ${ }^{5}$ Centre for Evaluation of \\ Medicines, St Joseph's Healthcare \\ Hamilton, Hamilton, Ontario, Canada; \\ ${ }^{6}$ Department of Family Medicine, \\ Faculty of Health Sciences, McMaster \\ University, Hamilton, Ontario, Canada
}

Correspondence: Jean-Eric Tarride, PATH Research Institute, 25 Main Street West, Suite 2000, Hamilton, ON L8P I HI, Canada

$\mathrm{Tel}+\mathrm{I} 9055237284$ ext 5282

Fax + I 9055220568

Email tarride@mcmaster.ca
Background: Obesity is today's principal neglected public health problem, as a rising proportion of adults will succumb to the medical complications of obesity. However, little is known about the burden of obesity in adults living in Ontario.

Objectives: To present an overview of the human and economic burden associated with BMI categories in Ontario, Canada, in terms of socio-demographics, comorbidities, health-related quality of life (HRQoL) and costs associated with hospitalization, same day procedures and physician visits.

Methods: The records of all Ontarians who participated in the Canadian Community Health Survey (CCHS), cycle 1.1 and provided consent to data linkage were linked to three administrative databases. Socio-demographic variables, medical characteristics, HRQoL, one year hospitalization, day procedure and physician costs were described per BMI category. Regression analyses were conducted to identify predictors of medical characteristics, HRQoL and costs.

Results: More than 50\% of adult participants were either overweight or obese in 2000/2001. Obese adults, and to a lesser extent overweight adults, were more likely to report physiciandiagnosed comorbid conditions, to use medications, and to have a lower HRQoL. After covariate adjustment, the hospitalization and physician costs were respectively $40 \%$ and $22 \%$ higher among obese and overweight adults than among normal-weight adults. No statistical cost differences were observed between normal and underweight individuals or between normal and overweight individuals. HRQoL was significantly lower in underweight and obese adults when compared to normal-weight individuals.

Conclusions: Due to the large human and economic burden associated with under- or excessweight, policies promoting healthy weight should remain a priority for governments and employers.

Keywords: health-related quality of life, costs, body mass index categories, Ontario

\section{Introduction}

Over the last two decades, several studies have documented the economic burden of overweight and obesity in Canada. The direct costs of overweight (Body Mass Index [BMI] 25-29 kg/m²) and obesity $\left(B M I \geq 30 \mathrm{~kg} / \mathrm{m}^{2}\right)$ in 2006 were estimated at approximately $\$ 2$ billion and $\$ 4$ billion respectively (all values are Canadian dollars), a figure that represents $4.1 \%$ of the total healthcare expenditure in Canada. Indirect costs accounted for $\$ 5$ billion. ${ }^{1}$ While this study or other similar Canadian burden of illness studies using national accounts ${ }^{2,3}$ provide valuable information, these studies did not provide a breakdown of health care utilization/medical costs per BMI or any information on the human burden associated with obesity (eg, health status, healthrelated quality of life [HRQoL]). 
Using a different approach, the physician costs associated with overweight and obesity in Ontario have been documented by linking a population health survey with physician billings. ${ }^{4}$ Compared to women and men with a normal body weight, annual physician costs were found to be $18 \%$ higher in obese women ( $\$ 682$ versus $\$ 577$ ) and 15\% higher in obese men ( $\$ 475$ versus $\$ 414)$. No differences in physician costs were observed between normal-weight adults and overweight adults. Although this study provided an estimate of the physician costs per BMI level in Ontario using patient-level data, it is currently unknown whether costs associated with hospitalizations or day procedures differ between BMI levels. This is important as hospital costs represent an important component of healthcare costs. Again, this study did not present any information on the medical characteristics and the HRQoL of this population, thus offering an incomplete picture of the burden of obesity in Ontario. While other Canadian studies have shown that obese individuals were more likely to have medical conditions, to report a worse HRQoL than normal-weight individuals and to consume more medical resources, ${ }^{5-7}$ these studies were limited as they did not include the elderly ${ }^{6}$ or all BMI categories (eg, overweight). ${ }^{7}$ There is also a lack of data on the Canadian costs associated with being underweight. ${ }^{8}$

To provide a broader assessment of differences between BMI categories, the objectives of this study were to describe the Ontario adult population per BMI category (eg, sociodemographic variables, HRQoL and costs) and to determine whether BMI levels were predictors of individuals' medical characteristics, HRQoL and costs including physician costs. By linking population survey and administrative data, this study improves on previous Canadian studies as it presents a detailed assessment of the human (HRQoL) and economic (eg, inpatient costs) burden associated with different BMI categories in Ontario. It also presents new and previously unavailable information on the health status associated with BMI (eg, HRQoL and cost data on underweight patients) and on the day procedure and hospitalization costs associated with BMI categories in adults living in Ontario.

\section{Materials and methods}

\section{Study population and setting}

This study included records of all Ontarians older than 18 years of age who participated in the Canadian Community Health Survey (CCHS), cycle 1.1 (2000/2001) and provided consent to data linkage with administrative databases. Three administrative databases were used to document the costs associated with hospitalizations, day procedures and physicians' services in Ontario. The Discharge Abstract Database (DAD), Inpatient (DAD-IP) and DAD-Day Procedure (DAD-DP) were used for hospitalizations (inpatient acute, chronic and rehabilitation care) and day procedures. These databases are maintained by the Canadian Institute of Health Information (CIHI) and provide patient-level information on patient (eg, age) and admission (eg, length of stay) characteristics. The Ontario Health Insurance Program (OHIP) database was used to identify all claims made by physicians paid through fee-for-service mechanisms (approximately 95\% of all Ontario physicians) and for diagnostic or laboratory tests conducted outside of hospitals. OHIP is maintained by the Ontario Ministry of Health and Long-Term Care (MOHLTC), which provided three fiscal years of data (1999/2000, 2000/2001 and 2001/2002). The record linkage between CCHS and the administrative databases was conducted by Statistics Canada.

\section{Survey data}

The BMI calculated by Statistics Canada (excluding pregnant women) was used to classify respondents according to their self-reported BMI: underweight (BMI $<18.5 \mathrm{~kg} / \mathrm{m}^{2}$ ); normal-weight $\left(18.5 \leq\right.$ BMI $\left.<24.9 \mathrm{~kg} / \mathrm{m}^{2}\right)$; overweight $\left(25 \leq \mathrm{BMI}<29.9 \mathrm{~kg} / \mathrm{m}^{2}\right.$; and obese $\left(\mathrm{BMI} \geq 30 \mathrm{~kg} / \mathrm{m}^{2}\right)$. Using self-reported information from CCHS 1.1, the population was described by BMI levels in terms of socio-demographic (eg, age) and medical characteristics (eg, comorbid conditions) as well as by health status (excellent, very good, good, fair and poor health) and Health Utility Index (HUI) score. ${ }^{9}$ While negative values can be observed, the HUI score generally ranges from 0 to 1 where 0 represents death and 1 represents being in excellent health. The HUI scores calculated by Statistics Canada were used in the analyses.

\section{Administrative data}

The administrative databases linked to CCHS data were used to estimate the costs associated with hospitalizations, day procedures and physician use for each BMI category. Using DAD-IP, inpatient hospital stays were assigned cost values using the resource intensity weight (RIW) (a standard costing methodology developed by CIHI) recorded for each admission which was multiplied by the average cost per RIW. The same approach was applied to same day procedures using DAD-DP. The OHIP database was used to identify the costs associated with physicians and nonhospital laboratories. The cost data analysis was conducted over a 1 year period covering the period of 6 months prior and 6 months after the CCHS interview date. The choice of 
this time horizon was dictated by two reasons. First, almost half of CCHS interviews occurred between April and October 2001. As such, since the latest fiscal year available for the administrative data was April 1, 2001 to March 31, 2002, the majority of respondents had less than 1 year of data following the interview. In addition, as weight and BMI may change over time, estimates of costs over a longer time period (eg, 2 years) before or after the date of CCHS interview may be misleading.

\section{Statistical analyses}

Socio-demographic variables, medical characteristics and health status were summarized using means, standard deviations and frequencies. Due to the multistage stratified sampling design of CCHS, Statistics Canada CCHS sample weights were applied to ensure that the results were representative of the Ontario population. Significant differences between each pair of BMI categories were assessed using Tukey's multiple comparison test for continuous variables ${ }^{10}$ and a modification of this method for testing for differences in proportions between multiple groups. ${ }^{11}$

Logistic regressions were used to determine the odds ratio of having comorbid conditions, taking a medication, being hospitalized or having a day surgery. The HUI utility data was analyzed with a Tobit model ${ }^{12}$ as utility data is often skewed with a ceiling effect at 1 . The cost data was modeled using a two-part model, ${ }^{13,14}$ a commonly used approach for the analysis of obesity cost data. ${ }^{4,15-17}$ This type of model is often used to take into account that a proportion of the population does not use medical resources (eg, no physician visits or hospitalizations in a given period of time) and therefore do not incur any costs. In Hurdle models, the data is modeled using two processes. First, a logistic regression is used to calculate the probability of observing a cost. A generalized linear model defined by a logarithmic link function with a gamma distribution is then used to model the positive costs. To generate the expected cost, the probability of having any use and the expected cost conditional on use were multiplied together. The excess costs of being underweight, overweight or obese versus normal-weight were also determined. To exploit the richness of the sampling of CCHS 1.1, bootstrap techniques were used to generate confidence intervals associated with the expected costs and excess costs versus normal-weight individuals. $^{4}$

The covariates included in the regression analyses were age, gender, physical activity (active, moderately active, and inactive), BMI level (normal-weight, underweight, overweight, and obese), personal income, and smoking status (current, former or never smoker). These covariates were chosen as they were previously used in other similar studies $^{4,6}$ as they are indirectly related to obesity and costs. The level of statistical significance was set at 0.05 a priori for all statistical tests and regressions. When the number of observations per cell was less than 10 , the information was not reported. Persons with total expenditures exceeding the 99th percentile were excluded from the cost analysis to reduce the influence of high-cost outliers. The costs analyses were conducted for the hospitalizations, day procedures, physician costs and for the total combined costs (hospital costs + day procedure costs + physician costs or "total costs"). To get a better understanding of the structure of the total costs, sub-group analyses were conducted per gender and age groups.

\section{Results}

The sample population for all analyses was based on 28,797 adult individuals who participated in CCHS 1.1, provided consent to data linkage, and who also had a BMI recording in the CCHS. More than half of the adult population of Ontario was overweight $(35 \%)$ or obese $(17 \%)$. The proportion of adults overweight or obese increased with age while the proportion of underweight individuals decreased in older adults.

\section{Socio-demographic characteristics}

The mean age of all adults was 45 years (SD: 17.2) and almost $30 \%$ of the population was 60 years of age and older. Statistical differences between all BMI categories were observed in terms of age, gender, marital status, ethnicity and physically activity status. In terms of education, obese individuals were statistically less likely to have a university degree compared to normal-weight individuals (29.6\% versus $42.1 \%$, respectively) (Table 1). Overweight adults had statistically higher personal and household incomes compared to other BMI categories. Table 1 presents the details.

\section{Medical characteristics}

Approximately $64 \%$ of adults with a normal-weight reported being diagnosed with at least one medical condition and $21 \%$ reported three or more medical conditions. Even after covariate adjustment, obese individuals were twice as likely to have reported three or more physician-diagnosed medical conditions compared to normal-weight individuals (Table 2). The probability of reporting a medical condition also increased with age, being female, being overweight 
Table I Socio-demographic characteristics in adults (18 years and older) ${ }^{\mathrm{a}}$

\begin{tabular}{|c|c|c|c|c|}
\hline & $\begin{array}{l}\text { Normal-weight } \\
\mathrm{n}=\mid \mathrm{I}, 828\end{array}$ & $\begin{array}{l}\text { Underweight } \\
n=2075\end{array}$ & $\begin{array}{l}\text { Overweight } \\
n=10,033\end{array}$ & $\begin{array}{l}\text { Obese } \\
n=486\end{array}$ \\
\hline Age (SD) & $43.1(18.1)$ & $39.9(20.4)$ & $47.5(16.0)$ & $47.4(14.5)$ \\
\hline $18-39$ & $40.9 \%$ & $50.7 \%$ & $29.8 \%$ & $29.4 \%$ \\
\hline $40-59$ & $32.4 \%$ & $23.7 \%$ & $38.3 \%$ & $42.7 \%$ \\
\hline 60 and over & $26.6 \%$ & $25.6 \%$ & $31.9 \%$ & $27.9 \%$ \\
\hline \multicolumn{5}{|l|}{ Gender } \\
\hline Male & $46.1 \%$ & $28.3 \%$ & $58.8 \%$ & $52.5 \%$ \\
\hline Female & $53.9 \%$ & $71.7 \%$ & $41.2 \%$ & $47.5 \%$ \\
\hline \multicolumn{5}{|l|}{ Marital status } \\
\hline Married and common law (living with partner) & $61.0 \%$ & $51.8 \%$ & $71.4 \%$ & $66.9 \%$ \\
\hline $\begin{array}{l}\text { Single, never married, and other } \\
\text { (widowed, separated, divorced) }\end{array}$ & $39.0 \%$ & $48.2 \%$ & $28.6 \%$ & $33.1 \%$ \\
\hline \multicolumn{5}{|l|}{ Place of birth } \\
\hline Born in Canada & $67.0 \%$ & $63.5 \%$ & $69.9 \%$ & $74.6 \%$ \\
\hline Born elsewhere & $33.0 \%$ & $36.5 \%$ & $30.1 \%$ & $25.4 \%$ \\
\hline \multicolumn{5}{|l|}{ Education } \\
\hline University degree & $42.1 \%$ & $39.3 \%$ & $38.5 \%$ & $29.6 \%$ \\
\hline No University degree & $57.9 \%$ & $60.7 \%$ & $61.5 \%$ & $70.4 \%$ \\
\hline \multicolumn{5}{|l|}{ Smoking Status } \\
\hline Current smoker & $27.5 \%$ & $30.1 \%$ & $24.7 \%$ & $24.2 \%$ \\
\hline Former smoker & $35.2 \%$ & $29.7 \%$ & $42.2 \%$ & $43.5 \%$ \\
\hline Never smoked & $37.3 \%$ & $40.2 \%$ & $33.1 \%$ & $32.3 \%$ \\
\hline \multicolumn{5}{|l|}{ Physical activity } \\
\hline Active & $22.7 \%$ & $21.7 \%$ & $21.1 \%$ & $13.5 \%$ \\
\hline Moderate & $24.1 \%$ & $19.5 \%$ & $23.7 \%$ & $22.2 \%$ \\
\hline Inactive & $53.2 \%$ & $58.8 \%$ & $55.2 \%$ & $64.5 \%$ \\
\hline \multicolumn{5}{|l|}{ Working status } \\
\hline Employed & $86.6 \%$ & $87.9 \%$ & $84.1 \%$ & $84.2 \%$ \\
\hline Not employed & $13.4 \%$ & $12.1 \%$ & $15.9 \%$ & $15.8 \%$ \\
\hline \multicolumn{5}{|l|}{ Income } \\
\hline Total personal income & $\$ 26,514$ & $\$ 34,614$ & $\$ 40,674$ & $\$ 34,777$ \\
\hline Household income & $\$ 67,903$ & $\$ 62,005$ & $\$ 69,848$ & $\$ 63,134$ \\
\hline
\end{tabular}

Notes: ${ }^{a}$ Based on complete data except for education $(n=16,702$ out of 28,797$)$ and working status ( $n=24,564$ out of 28,797$)$; bold indicates statistical significance (ie, $P<0.05$ ) compared to normal-weight.

or being a current or former smoker. Obese individuals were three to four times more likely to have high blood pressure and diabetes than normal-weight individuals and twice as likely to report arthritis/rheumatism, urinary incontinence or fibromyalgia. Other differences between BMI levels were also observed (Table 2). For example, underweight individuals were statistically less likely to report diabetes, arthritis, and high blood pressure than normal-weight adults, and more likely to report bowel disorders, emphysema or COPD, chronic fatigue syndrome, and epilepsy.

Consistent with the reporting of medical conditions, the odds of using blood pressure medications, diuretics, or diabetes medications were three times higher among obese individuals than normal-weight individuals. Obese adults were also twice as likely to use steroids or cardiac medications as normal-weight individuals (Table 3). Overweight individuals also took more medications than normal-weight adults, but to a lesser extent than obese adults. Underweight individuals had lower use-patterns of medications for the treatment of conditions such as diabetes and hypertension than normal-weight individuals.

\section{Health-related quality of life and self-reported health}

The HUI scores associated with underweight $(0.85 \pm 0.25)$ and obese individuals $(0.83 \pm 0.23)$ were statistically lower than normal-weight individuals $(0.89 \pm 0.19)$ (Table 4$)$. The HUI scores decreased with age for all BMI categories. For the 60 and over group, underweight individuals had a significantly lower utility than other adults. A Tobit regression indicated that increased age, being female, being a current or a former smoker, being underweight, overweight or obese were significantly associated with a decrease in the HUI utility scores while personal income and being physically active were significantly associated 
Table 2 Medical characteristics for adults with a normal BMI level (I8 years and older) and odds ratio (confidence intervals) by BMI levels (reference: normal-weight) ${ }^{\mathrm{b}}$

\begin{tabular}{|c|c|c|c|c|}
\hline & $\begin{array}{l}\text { Normal-weight } \\
\text { (\%) }\end{array}$ & $\begin{array}{l}\text { OR and Cls underweight } \\
\text { versus normal-weight }\end{array}$ & $\begin{array}{l}\text { OR and Cls overweight } \\
\text { versus normal-weight }\end{array}$ & $\begin{array}{l}\text { OR and Cls obese } \\
\text { versus normal-weight }\end{array}$ \\
\hline At least I medical condition & $64.1 \%$ & $0.92(0.8,1.0)$ & $1.2(1.1,1.3)$ & $1.8(1.6,1.9)$ \\
\hline$\geq 3$ medical conditions & $21.1 \%$ & $1 . I(1.0,1.2)$ & $1.3(1.2,1.4)$ & $2.1(2.0,2.3)$ \\
\hline \multicolumn{5}{|l|}{ Specific medical conditions } \\
\hline Allergies other than food & $28.0 \%$ & $1.0(0.9,1.1)$ & I.I (I.0, I.I) & $1.2(I . I, I .3)$ \\
\hline Back problems & $17.6 \%$ & $\mathrm{I} .0(0.8, \mathrm{I} . \mathrm{I})$ & $1.2(I . I, I .3)$ & $1.5(1.4,1.6)$ \\
\hline Arthritis/rheumatism & $15.1 \%$ & $0.8(0.7,0.9)$ & $1.3(1.3,1.5)$ & $2.2(2.0,2.4)$ \\
\hline High blood pressure & $9.9 \%$ & $0.7(0.5,0.8)$ & $1.8(1.7,2.0)$ & $3.6(3.3,4.0)$ \\
\hline Other chronic condition & $11.7 \%$ & $1.0(0.9,1.2)$ & I.I (I.0, I.2) & $1.3(1.2,1.5)$ \\
\hline Migraine & $9.4 \%$ & $1.0(0.9,1.2)$ & $1.2(1.1,1.3)$ & $1.5(1.4,1.7)$ \\
\hline Asthma & $9.2 \%$ & I.I $(1.0,1.4)$ & $1.3(1.1,1.4)$ & $1.8(1.6,2.0)$ \\
\hline Food allergies & $7.6 \%$ & I.I $(0.9,1.3)$ & $\mathrm{I} .0(0.9, \mathrm{I} . \mathrm{I})$ & I.0 (0.9, I.2) \\
\hline Heart disease & $5.1 \%$ & $1.0(0.8,1.2)$ & $1.2(1.0,1.3)$ & I.7 (I.5, 2.0) \\
\hline Thyroid & $4.4 \%$ & $0.9(0.7, I . I)$ & $1.3(1.1,1.4)$ & $1.7(1.5,1.9)$ \\
\hline Diabetes & $2.9 \%$ & $0.6(0.4,0.9)$ & $1.8(1.6,2.0)$ & $3.8(3.3,4.3)$ \\
\hline Cataracts & $4.1 \%$ & $0.9(0.7,1.1)$ & I.I (I.0, I.3) & $1.3(1.1,1.6)$ \\
\hline Stomach/intestinal ulcers & $2.8 \%$ & $1.2(0.9,1.5)$ & $1.2(1.0,1.4)$ & $1.6(1.4,1.9)$ \\
\hline Bronchitis & $2.4 \%$ & $1.3(1.0,1.6)$ & $\mathrm{I} .0(0.9, \mathrm{I} .2)$ & $2.0(1.7,2.3)$ \\
\hline Bowel disorder & $2.5 \%$ & $1.3(1.0,1.7)$ & $1.2(1.0,1.4)$ & $1.5(1.2,1.8)$ \\
\hline Urinary incontinence & $2.1 \%$ & $1.2(0.9,1.6)$ & $1.3(I .1,1.5)$ & $2.4(2.0,2.9)$ \\
\hline Cancer & $1.7 \%$ & $1.0(0.7,1.4)$ & $1.0(0.8,1.2)$ & $1.2(0.9,1.4)$ \\
\hline Glaucoma & $1.3 \%$ & $1.2(0.8,1.6)$ & I.I $(0.9,1.3)$ & I.I $(0.9,1.5)$ \\
\hline Fibromyalgia & $1.2 \%$ & I.0 $(0.7,1.5)$ & $1.4(1.1,1.8)$ & $2.3(1.8,2.9)$ \\
\hline Emphysema or COPD & $1.1 \%$ & $2.6(1.8,3.7)$ & $0.9(0.7, \mathrm{I} . \mathrm{I})$ & $1.6(1.2,2.1)$ \\
\hline Effects of a stroke & $1.3 \%$ & I.2 $(0.8,1.8)$ & $0.9(0.7,1.2)$ & $1.5(1.2,2.0)$ \\
\hline Chronic fatigue syndrome & $0.9 \%$ & $1.5(1.0,2.3)$ & $1.0(0.7,1.3)$ & $1.7(1.3,2.4)$ \\
\hline Epilepsy & $0.5 \%$ & $1.8(1.1,2.9)$ & $0.8(0.6,1.2)$ & $1.6(1.1,2.4)$ \\
\hline Alzheimer's disease & $0.3 \%$ & $1.0(0.3,2.9)$ & $0.7(0.3,1.3)$ & $0.9(0.4,2.0)$ \\
\hline Multiple sclerosis & $0.3 \%$ & $1.7(0.9,3.4)$ & $0.5(0.3,1.0)$ & $0.7(0.4,1.4)$ \\
\hline
\end{tabular}

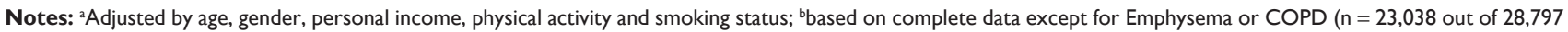
respondents); bold indicates statistical significance (ie, $P<0.05$ ) compared to normal weight.

Abbreviations: $\mathrm{BMI}$, body mass index; OR, odds ratio; $\mathrm{Cl}$, confidence interval; $\mathrm{COPD}$, chronic obstructive pulmonary disease.

with an increase in HUI utility scores. Self-reported health status was significantly worse for obese individuals with more than $20 \%$ of obese adults reporting having fair or poor health, compared to $10 \%$ for normal-weight adults (Table 4). Approximately $15 \%$ of obese adults reported a deterioration of their health status versus $10 \%$ for normalweight individuals.

\section{Economic burden associated with $\mathrm{BMI}$ in adults}

Table 5 presents the mean 1 year adjusted costs associated with hospitalizations, day procedures and physician services as well as the stratified analyses for total costs. In terms of hospitalizations, obese individuals have statistically higher annual costs than normal-weight individuals (\$233 versus $\$ 166$ or a $40 \%$ difference). Obese aindividuals also have significantly higher physician and total costs (ie, combined hospital, day procedure, and physician costs) than normal-weight adults (Table 5). No statistical cost differences were observed between overweight and normalweight individuals or between underweight and normalweight individuals even when the total cost analyses were stratified by gender and age groups (Table 5). However, the total costs among obese men were not statistically different from the total costs among normal-weight men, while this difference was statistically significant among women. Specifically, the results of the hurdle models used to estimate the total costs by gender indicated that being an obese man compared to a normal-weight man was not a significant factor in modeling the first (probability of observing a cost) and the second (modeling costs conditional on use) part of the model. In contrast, obese women had significantly higher costs than normal-weight women once a cost had been incurred. In all regressions by gender, age was always a statistically significant variable at any stage of the hurdle model (ie, modeling both the probability of incurring a cost and modeling costs once a cost has been incurred) while being physically active statisti- 
Table 3 Percentage of adults with specific medications and odds ratio (confidence intervals) by BMI levels (reference: normal-weight)

\begin{tabular}{|c|c|c|c|c|}
\hline & $\begin{array}{l}\text { Normal-weight } \\
\text { (\%) }\end{array}$ & $\begin{array}{l}\text { OR and Cls underweight } \\
\text { versus normal-weight }\end{array}$ & $\begin{array}{l}\text { OR and Cls overweight } \\
\text { versus normal-weight }\end{array}$ & $\begin{array}{l}\text { OR and Cls obese } \\
\text { versus normal-weight }\end{array}$ \\
\hline Pain relievers & $67.9 \%$ & $0.8(0.7,0.9)$ & I.I (I.0, I.2) & $1.2(1.1,1.4)$ \\
\hline Birth control pills & $21.3 \%$ & I.I $(0.9, \mathrm{I} .3)$ & $0.9(0.7,1.0)$ & $0.9(0.7,1.1)$ \\
\hline Cough/cold & $18.0 \%$ & $0.9(0.8,1.1)$ & I.I (I.0, I.2) & $1.2(1.1,1.4)$ \\
\hline Other & $15.3 \%$ & $\mathrm{I} .0(0.9, \mathrm{I} .2)$ & I.I (I.0, I.2) & $1.4(1.3,1.6)$ \\
\hline Hormones for menopause & $13.9 \%$ & $0.8(0.6,0.9)$ & $1.2(1.0,1.3)$ & I.I $(0.9,1.3)$ \\
\hline Blood pressure & $9.4 \%$ & $0.7(0.6,0.8)$ & $1.8(1.6,2.0)$ & $3.5(3.1,3.9)$ \\
\hline Stomach & $10.4 \%$ & $1.0(0.8,1.1)$ & $1.3(1.2,1.4)$ & $1.6(1.4,1.8)$ \\
\hline Allergy medicine & $9.4 \%$ & $0.9(0.7,1.1)$ & $1.0(0.9,1.1)$ & I.I (I.0, I.3) \\
\hline Penicillin/other & $8.0 \%$ & $1.2(1.0,1.4)$ & $1.0(0.9,1.1)$ & $1.3(1.1,1.5)$ \\
\hline Asthma medications & $5.5 \%$ & $1.3(1.1,1.6)$ & $1.2(1.1,1.4)$ & $1.8(1.6,2.1)$ \\
\hline Codeine/demerol/morphine & $5.5 \%$ & $1.3(1.1,1.6)$ & $1.2(1.1,1.4)$ & $1.7(1.4,1.9)$ \\
\hline Medicine for the heart & $4.9 \%$ & $\mathrm{I} .0(0.8, \mathrm{I} .3)$ & $1.2(1.1,1.4)$ & $2.0(1.7,2.3)$ \\
\hline Antidepressants & $5.0 \%$ & $1.0(0.8,1.2)$ & $1.2(1.1,1.4)$ & $1.8(1.6,2.1)$ \\
\hline Sleeping pills & $4.8 \%$ & $1.3(1.0,1.5)$ & $1.0(0.9,1.1)$ & I.I $(0.9,1.2)$ \\
\hline Diuretics & $3.6 \%$ & $0.8(0.6,1.1)$ & $1.5(1.3,1.7)$ & $3.3(2.9,3.9)$ \\
\hline Thyroid & $4.1 \%$ & $0.8(0.6,1.0)$ & $1.2(1.0,1.4)$ & $1.7(1.4,1.9)$ \\
\hline Laxatives & $3.9 \%$ & I.I $(0.9$, I.4) & $0.9(0.8,1.1)$ & I.I $(0.9$, I.3) \\
\hline Control diabetes & $1.8 \%$ & $0.4(0.3,0.8)$ & I.8 (1.5, 2.2) & $3.7(3.1,4.6)$ \\
\hline Tranquilizers & $2.7 \%$ & $1.7(1.3,2.2)$ & I.I $(0.9,1.3)$ & $1.5(1.2,1.8)$ \\
\hline Steroids & $1.3 \%$ & I.2 $(0.8,1.8)$ & $1.2(1.0,1.6)$ & $2.0(1.6,2.7)$ \\
\hline Insulin & $0.5 \%$ & $0.9(0.5,1.8)$ & $1.9(1.4,2.6)$ & $3.3(2.4,4.6)$ \\
\hline
\end{tabular}

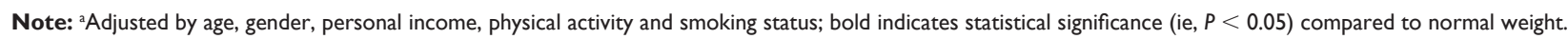

Abbreviations: $\mathrm{BMI}$, body mass index; OR, odds ratio; $\mathrm{Cl}$, confidence interval.

cally decreased the costs once a cost has been incurred. For all BMI categories, physician costs were larger than hospitalization costs (Table 5).

Overall, $8 \%$ of all adults were hospitalized. When compared to the normal-weight group, underweight (OR 1.3; CIs: 1.1, 1.6) and obese adults (OR 1.3; CIs: 1.2, 1.5) were more likely to be hospitalized after covariate adjustment. While increased age and being female also increased the probability of being hospitalized, being overweight and personal income were not significant variables. The second part of the hurdle model used for the analysis of hospitalization costs indicated that, once hospitalized and after covariate adjustment, the expected costs incurred by underweight $(\$ 6,288$; SD: $\$ 1,326)$, overweight $(\$ 6,035$; SD: $\$ 1,033)$ and obese $(\$ 6,413$, SD: $\$ 1,002)$ individuals were not statistically different from the costs associated with the normal-weight group $(\$ 5,846 ;$ SD: $\$ 1,135)$. When analyzing the probability of having a day procedure or explaining costs conditional on use, being obese was a significant variable for the two stages of the hurdle model while underweight and overweight were

Table 4 Health-related quality of life for adults (18 years and older) ${ }^{\mathrm{a}}$

\begin{tabular}{|c|c|c|c|c|}
\hline & Normal-weight & Underweight & Overweight & Obese \\
\hline Mean (SD) HUI3 utility index & $0.89(0.19)$ & $0.85(0.25)$ & $0.87(0.19)$ & $0.83(0.23)$ \\
\hline $18-39$ & $0.91(0.17)$ & $0.90(0.19)$ & $0.91(0.16)$ & $0.89(0.19)$ \\
\hline $40-59$ & $0.88(0.19)$ & $0.85(0.24)$ & $0.88(0.19)$ & $0.82(0.24)$ \\
\hline 60 and over & $0.80(0.22)$ & $0.67(0.31)$ & $0.80(0.21)$ & $0.74(0.24)$ \\
\hline \multicolumn{5}{|l|}{ Self-reported health } \\
\hline Excellent/very good/good & $89.7 \%$ & $85.8 \%$ & $87.7 \%$ & $78.7 \%$ \\
\hline Fair/poor & $10.4 \%$ & $14.2 \%$ & $12.3 \%$ & $21.2 \%$ \\
\hline \multicolumn{5}{|l|}{ Compared to one year ago } \\
\hline Much better/somewhat/about same & $89.3 \%$ & $88.0 \%$ & $88.3 \%$ & $84.4 \%$ \\
\hline Somewhat worse/much worse now than I year ago & $10.7 \%$ & $11.9 \%$ & $11.7 \%$ & $15.6 \%$ \\
\hline \multicolumn{5}{|l|}{ Did something to improve health over last year } \\
\hline Yes & $52.5 \%$ & $48.9 \%$ & $54.6 \%$ & $55.5 \%$ \\
\hline No & $47.5 \%$ & $51.1 \%$ & $45.4 \%$ & $44.5 \%$ \\
\hline
\end{tabular}

Notes: ${ }^{a}$ Based on complete data except for the last question "Did something to improve health over the last year" ( $n=27,265$ out of 28,797 respondents); bold indicates statistical significance (ie, $P<0.05$ ) compared to normal-weight.

Abbreviation: SD, standard deviation. 
Table 5 One year physician, hospitalization, day procedure and total costs ${ }^{\mathrm{a}}$ according to BMl categories and cost differences (reference: normal-weight) (mean and $95 \%$ confidence intervals) ${ }^{\mathrm{b}}$

\begin{tabular}{|c|c|c|c|c|}
\hline & Normal-weight & Underweight & Overweight & Obese \\
\hline Hospitalisation costs & $\$ 166.3(\$ 144.3, \$ 190.3)$ & $\$ 206.1(\$ 150.3, \$ 270.6)$ & $\$ 165.2(\$ 140.0, \$ 190.5)$ & $\$ 233.3(\$ 192.7, \$ 276.3)$ \\
\hline Cost difference & Reference group & $\$ 39.8(-\$ 22.5, \$ 110.6)$ & $-\$ 1.1(-\$ 35.3, \$ 29.0)$ & $\$ 67.0(\$ 23.5, \$ 1 \mid 4.2)$ \\
\hline Day procedure costs & $\$ 57.6(\$ 49.9, \$ 66.7)$ & $\$ 54.2(\$ 39.7, \$ 69.1)$ & $\$ 60.1(\$ 53.6, \$ 67.0)$ & $\$ 67.3(\$ 58.1, \$ 76.8)$ \\
\hline Cost difference & Reference group & $-\$ 3.4(-\$ 18.8, \$ 13.0)$ & $\$ 2.5(-\$ 8.6, \$ 12.9)$ & $\$ 9.7(-\$ 0.9, \$ 20.7)$ \\
\hline Physician costs & $\$ 476.8(\$ 457.6, \$ 498.4)$ & $\$ 487.8(\$ 447.1, \$ 530.3)$ & $\$ 463.4(\$ 44 I .8, \$ 487.3)$ & $\$ 584.9(\$ 544.3, \$ 630.8)$ \\
\hline Cost difference & Reference group & $\$ 11.0(-\$ 34.3, \$ 56.0)$ & $-\$ 13.4(-\$ 43.3, \$ 14.1)$ & $\$ 108.1(\$ 63.9, \$ 155.4)$ \\
\hline Total costs ${ }^{\mathrm{a}}$ & $\$ 708.0(\$ 668.2, \$ 752.4)$ & $\$ 746.0(\$ 652.0, \$ 834.6)$ & $\$ 690.3(\$ 648.2, \$ 736.4)$ & $\$ 884.1$ (\$806.I, \$953.8) \\
\hline Cost difference & Reference group & $\$ 38.0(-\$ 57.7, \$ 138.9)$ & $-\$ 17.7(-\$ 74.2, \$ 38.4)$ & $\$ 176.1(\$ 88.3, \$ 252.8)$ \\
\hline Total costs: male & $\$ 600.1(\$ 543.3, \$ 659.8)$ & $\$ 691.3(\$ 521.6, \$ 911.5)$ & $\$ 540.3(\$ 497.3, \$ 581.5)$ & $\$ 671.2(\$ 585.3, \$ 765.5)$ \\
\hline Cost difference & Reference group & $\$ 9 \mid .2(-\$ 97.4, \$ 3 \mid 2.6)$ & $-\$ 59.8(-\$ 133.4, \$ 14.1)$ & $\$ 7 I . I(-\$ 36.1, \$ 178.3)$ \\
\hline Total costs: female & $\$ 812.9(\$ 755.2, \$ 863.3)$ & $\$ 821.4(\$ 7 \mid 0.1, \$ 944.6)$ & $\$ 849.4(\$ 781.6, \$ 932.4)$ & $\$ 1121.9(\$ 1013.4, \$ 1243.5)$ \\
\hline Cost difference & Reference group & $\$ 8.5(-\$ 105.1, \$ 140.2)$ & $\$ 36.5(-\$ 45.0, \$ 128.3)$ & $\$ 309.0(\$ 185.3, \$ 441.4)$ \\
\hline Total costs: & $\$ 424.3(\$ 389.2, \$ 460.0)$ & $\$ 404.1(\$ 338.8, \$ 480.5)$ & $\$ 436.4(\$ 394.3, \$ 483.4)$ & $\$ 506.3(\$ 435.1, \$ 586.6)$ \\
\hline \multicolumn{5}{|l|}{ Young adults (I8-39) } \\
\hline Cost difference & Reference group & $-\$ 20.2(-\$ 87.6, \$ 61.6)$ & $\$ 12.1(-\$ 50.9, \$ 71.4)$ & $\$ 82.0(\$ 2.0, \$ 176.1)$ \\
\hline Total costs: & $\$ 673.4(\$ 613.9, \$ 745.7)$ & $\$ 810.4(\$ 599.6, \$ 1040.0)$ & $\$ 618.7(\$ 556.7, \$ 685.5)$ & $\$ 918.3(\$ 809.3, \$ 1051.2)$ \\
\hline \multicolumn{5}{|c|}{ Middle-age adults (40-59) } \\
\hline Cost difference & Reference group & $\$ 137.0(-\$ 79.1, \$ 382.0)$ & $-\$ 54.7(-\$ 137.7, \$ 33.6)$ & $\$ 244.9(\$ 108.9, \$ 393.1)$ \\
\hline Total costs: & $\$ 1230.5(\$ 1 \mid 23.1, \$ 1348.7)$ & $\$ 1201.9(\$ 975.8, \$ 1462.3)$ & $\$ 1282.9(\$ 1 \mid 62.9, \$ 1399.0)$ & $\$ 1476.1$ (\$1296.9, \$1665.7) \\
\hline \multicolumn{5}{|l|}{ Older adults $(60$ and + ) } \\
\hline Cost difference & Reference group & $-\$ 28.6(-\$ 268.4, \$ 220.1)$ & $\$ 52.4(-\$ 95.1, \$ 177.1)$ & $\$ 245.6(\$ 59.1, \$ 442.3)$ \\
\hline
\end{tabular}

Notes: ${ }^{\text {aTotal }}$ cost $=$ hospitalization costs + day procedure costs + physician costs; badjusted for personal income, smoking status, physical activity status, age and gender; all values are Canadian dollars; bold indicates statistical significance (ie, $P<0.05$ ) compared to normal weight.

not significant variables when compared to normal weight individuals.

\section{Discussion}

This study confirms that being obese is associated with a negative impact on health and higher health care costs for adults in Ontario. Specifically, obese adults were more likely to have a lower health-related quality of life (ie, 0.83 ) than normal-weight adults (ie, 0.89). As a reference, a difference in the HUI score of 0.05 between two groups is clinically significant. ${ }^{9}$ In addition, obese individuals were more likely to report more medical conditions, to take more medications and to incur higher costs due to hospitalizations and physician services. Although being overweight was not associated with an increased cost in our analyses, it should be noted that many overweight individuals will end up obese. For example, Canadian data have shown that almost a quarter of individuals who were overweight in 1994/95 had become obese by $2002 / 03$. In contrast, only $10 \%$ of overweight individuals in 1994-95 had a normal weight in 2002-03. ${ }^{18}$ As shown by CCHS 1.1, less than half of the Ontario adult population reported having a normal BMI in 2000/2001. It is therefore important that health systems consider broadly implementing programs that target both prevention (eg, in the community, school and workplace) as well as obesity treatment (eg, interdisciplinary primary care teams, and specialist services including bariatric surgery). We also observed no statistical significance difference between the total costs of obese and normal weight men while this difference was statistically significant for women. Two main factors may explain these results. In all regressions, women had significantly higher physician, day procedure and hospitalization costs than men once a cost has been incurred. In addition, women had a higher probability of being hospitalized or undergoing a day procedure than men. As such, programs targeted towards women may have greater potential for reducing costs associated with hospitalization, day procedures, and physicians. Similarly, in light of the magnitude of the cost differences between obese and normal weight group in the 40-59 year old age group, efforts should be put into targeting middleaged obese individuals. In this context, the workplace may be a suitable environment for the implementation of programs aimed at preventing obesity and its related complications (eg, diabetes, hypertension).

Recently two reviews on the burden of obesity have been published. The first ${ }^{19}$ reviewed 32 studies published between 1990 and June 2009 which evaluated the direct costs associated with obesity. Despite differences in study designs, all studies indicated that the financial burden of obesity was significant, accounting for between $0.7 \%$ and 
$2.8 \%$ of healthcare expenditures. This review also reported that the costs associated with obesity were estimated to be $30 \%$ higher than that of normal-weight individuals, which is consistent with our analyses (24\% for the total costs). Another recent review focussed on the direct medical cost of being overweight and obese in the US, based on 33 US studies published up to September 2009. Depending on which studies were included, the per-person excess costs associated with overweight ranged from \$298 to \$498 when compared to normal-weight BMI. In comparison, the incremental cost of obesity was estimated to range from $\$ 1630$ to $\$ 1723$. Based on these figures, the authors estimated that the direct medical cost of overweight and obesity accounted for almost $5 \%$ of the total US health expenditure, which is comparable to the most recent Canadian estimate (4.1\%). ${ }^{1}$ Although these two reviews provide invaluable information, it is difficult to directly compare the results of these studies with our study. With the exception of Janssen et $\mathrm{al}^{4}$ and Finkelstein ${ }^{20}$, no studies have been conducted in Canada using population survey data linked to patient-level administrative data.

The interpretation of our results was similar to Janssen et $\mathrm{al}^{4}$ despite the fact that we also analyzed hospital and day procedure costs. Specifically, the annual physician costs reported in our study were similar to those reported by Janssen et $\mathrm{al}^{4}$ even if Janssen et al used two years of fiscal data (2002 and 2003) to calculate an average physician cost per year while we used one year of data (ie, 6 months before and 6 months after the date of interview). Nonetheless, other calculations (data not shown) indicated that the physician costs associated with normal-weight and obese males in our study were \$408 and \$456, respectively, compared to \$414 and $\$ 475$ in Janssen's study. For women, the figures in our study were $\$ 549$ (normal-weight) and $\$ 726$ (obese), which compared to $\$ 577$ and $\$ 682$ in Janssen's study. ${ }^{4}$ Our analyses indicated that compared to normal-weight individuals, being overweight was not associated with a significant increase in hospitalization and day procedures costs. This finding was also observed by Janssen et $\mathrm{al}^{4}$ in their analysis of physician costs, and reported in a recent review of the direct costs of overweight and obesity in the US. ${ }^{21}$

Although an earlier record linkage conducted in Ontario calculated the additional physician costs for each unit increase in BMI at $\$ 8.90$, no information was provided on the physician costs per BMI category ${ }^{20}$ and therefore cannot be used to compare our results.

There are several limitations with our study that have to be considered when interpreting the results. First, the true costs associated with BMI categories are likely to be underestimated as drug costs, costs associated with other non-physician healthcare providers or indirect costs were not included as they were unavailable for analysis. Secondly, as found in several studies using surveys from Statistics Canada (eg, CCHS 1.1), ${ }^{4-7}$ individuals were classified according to self-reported BMI which may have introduced some bias in our estimates. Although other prevalence-based cost-ofillness studies have used measured BMI data from CCHS 2004, ${ }^{1,22}$ CCHS 1.1 was at the time of analysis the most recent CCHS data that could be linked with administrative databases at our Research Data Center. Nonetheless, the use of selfreported BMI may have introduced some bias in our estimate of the burden of obesity. As a reference, the measured prevalence of obesity in Canada was found to be $7 \%$ higher than the self-reported prevalence based on data from the 2005 CCHS in which height and weight were measured for a sub-sample of the survey. ${ }^{23}$ Differences of -8.5 BMI points (CI: -10.0 to -7.1 ) and of $7.4 \mathrm{BMI}$ points (CI: 6.0 to 8.8 ) between measured and self-reported BMI were reported for the normal and obese population, respectively. The sensitivity and specificity between self-reported and measured BMI for normal-weight people were 93\% (CI: 90-95) and 79\% (CI: 75-82), respectively. Sensitivity was lower for obese individuals $(63 \%)$ but the specificity was high (more than $95 \%$ ). Since very few respondents would report weight and height that would categorize them as being obese unless they were actually obese, our cost estimates for the obese population should closely reflect the costs associated with measured obesity. As such, the cost differences between the obese and normal-weight populations should also be reflective of the differences between these two categories based on measured BMI. Although numerical differences existed between measured versus self-reported BMI prevalence rates for the overweight category (ie, 1.9 BMI points), these differences were not statistically significant. In addition, our analyses did not show any statistically significant cost differences between normal-weight and overweight individuals. The interpretation of the results on quality of life associated with the BMI categories should not be impacted by the self-reported BMI as no statistically significant differences were observed in terms of activity limitation and self-perceived health when measured and selfreported BMI were compared. ${ }^{24}$ The most recent Canadian data $^{25}$ indicates that $26.1 \%$ and $23.3 \%$ of Canadian men and women were obese in 2008 after correcting for bias in selfreported estimates of BMI. In contrast, the proportions of obese men and women were respectively $18.5 \%$ and $16.1 \%$ in 2008 when using self-reported values, which was close 
to the CCHS 1.1 estimates (17\% of Ontarians estimated as obese). As such the costs that were reported in our study are probably higher.

Another important limitation is that BMI does not truly reflect the burden of obesity-related health risks. Thus, as recently demonstrated in several large US population samples, ${ }^{26,27}$ the Edmonton Obesity Staging System (EOSS), which classified overweight and obese individuals on a 5-point ordinal scale based on the presence of medical, mental and/or functional comorbidities, strongly predicted mortality, whereas BMI did not. Importantly, in this analysis, a considerable proportion (about 50\%) of individuals in the overweight range presented with obesity-related health problems (EOSS $\geq 1$ ), while a substantial number (about $30 \%$ ) of individuals with BMI $>30$ had no obesity-related health risks. Thus, our current analysis may underestimate the true health cost of excess weight to the health system.

Since we did not have access to drug claims data, we were not able to estimate drug costs per BMI category, which is an important limitation of our study. Although our analyses using the survey data indicated that excess weight was associated with an increase use of drugs, we were not able to estimate this cost. Our sample of respondents excluded respondents living in long-term care facilities, nursing homes or the armed forces. Finally, the data is almost ten years old and although the prevalence of obesity has increased over time, it is unknown whether the associations between obesity, its health outcomes and costs have changed over the last 10 years. Linking longitudinal population health surveys with several years of administrative data is an important avenue for future research.

Despite these limitations, this work helps to better understand the burden associated with BMI categories in Canada. In particular, it provides new and previously unavailable information on the costs associated with hospitalizations and day procedures per BMI category as well as new data on underweight adults (eg, HRQoL and costs). By exploiting the richness of survey data linked to administrative databases (using BMI from CCHS to calculate cost per BMI category using administrative database), the results emphasize the tremendous human and economic burden of underweight and obesity in Ontario. The data generated in this study, including costs and utilities per BMI level, can also be used to evaluate strategies aimed at decreasing or containing the burden of under- and excess-weight in Canada. Several authors of this paper have contributed to the development of the Ontario Diabetes Economic Model (ODEM) based on the United Kingdom Prospective Diabetes
Study combined with resource utilization, utilities and costs from Ontario. The ODEM is currently used by the Ontario Ministry of Health and Long-Term Care to evaluate diabetes management strategies. ${ }^{28}$ To better inform decision makers about the value of programs aimed at managing the burden of under and excess weight, the development of an obesity policy model is an important area of future research.

\section{Acknowledgments}

This investigator-initiated research was funded by an unrestricted grant from Pfizer Canada Inc. Drs Daria O'Reilly and Jean-Eric Tarride are recipients of Career Scientist Awards from the Ontario Ministry of Health and Long-term Care. The authors would also like to thank Dr Ian Janssen for providing the Bootstrap algorithm for the two-part model.

\section{Disclosure}

The authors report no conflicts of interest with this work.

\section{References}

1. Anis AH, Zhang W, Bansback N, Guh DP, Amarsi Z, Birmingham CL. Obesity and overweight in Canada: an updated cost-of-illness study. Obes Rev. 2010;11(1):31-40.

2. Birmingham CL, Muller JL, Palepu A, Spinelli JJ, Anis AH. The cost of obesity in Canada. CMAJ. 1999;160(4):483-488.

3. Katzmarzyk PT, Janssen I. The economic costs associated with physical inactivity and obesity in Canada: an update. Can J Appl Physiol. 2004; 29(1):90-115.

4. Janssen I, Lam M, Katzmarzyk PT. Influence of overweight and obesity on physician costs in adolescents and adults in Ontario, Canada. Obes Rev. 2009;10(1):51-57.

5. Chen Y, Jiang Y, Mao Y. Hospital admissions associated with body mass index in Canadian adults. Int J Obes (Lond). 2007;31(6):962-967.

6. Trakas K, Lawrence K, Shear NH. Utilization of health care resources by obese Canadians. CMAJ. 1999;160(10):1457-1462.

7. Trakas K, Oh PI, Singh S, Risebrough N, Shear NH. The health status of obese individuals in Canada. Int JObes Relat Metab Disord. 2001;25(5): 662-668.

8. Che J. Underweight Canadians. 11-008 Statistics Canada; 2002 Available from: http://www.statcan.gc.ca/kits-trousses/pdf/social/ edu04_0146a-eng.pdf. Accessed October 31, 2011.

9. Feeny D, Furlong W, Torrance GW, et al. Multiattribute and singleattribute utility functions for the health utilities index mark 3 system. Med Care. 2002;40(2):113-128.

10. Zar JH. Biostatistical Analysis. 4th Edition. New Jersey: Prentice-Hall; 1999.

11. Elliott AC, Reisch JS. Implementing a multiple comparison test for proportions in a 2xc crosstabulation in $S A S^{\circledR}$. Paper 204-31. Cary (NC): SAS Institute; 1999. Available from: http://www2.sas.com/proceedings/ sugi31/204-31.pdf. Accessed October 31, 2011.

12. Austin PC, Escobar M, Kopec JA. The use of the Tobit model for analyzing measures of health status. Qual Life Res. 2009;9(8):901-910.

13. Diehr P, Yanez D, Ash A, Hornbrook M, Lin DY. Methods for analyzing health care utilization and costs. Annu Rev Public Health. 1999;20: 125-144.

14. Mullahy J. Econometric modeling of health care costs and expenditures: a survey of analytical issues and related policy considerations. Med Care. 2009;47(7 Suppl 1):S104-S108. 
15. Finkelstein EA, Trogdon JG, Brown DS, Allaire BT, Dellea PS, Kamal-Bahl SJ. The lifetime medical cost burden of overweight and obesity: implications for obesity prevention. Obesity (Silver Spring). 2008;16(8):1843-1848.

16. Raebel MA, Malone DC, Conner DA, Xu S, Porter JA, Lanty FA. Health services use and health care costs of obese and nonobese individuals. Arch Intern Med. 2004;164(19):2135-2140.

17. Wee CC, Phillips RS, Legedza AT, et al. Health care expenditures associated with overweight and obesity among US adults: importance of age and race. Am J Public Health. 2005;95(1):159-165.

18. Statistics Canada. Obesity: a growing issue. In: Healthy today, healthy tomorrow? Findings from the National Population Health Survey [web page on the Internet]. Ottawa: Statistics Canada; 2005. Available from: http://www.statcan.gc.ca/pub/82-618-m/82-618-m2005003-eng.htm. Accessed November 10, 2011.

19. Withrow D, Alter DA. The economic burden of obesity worldwide: a systematic review of the direct costs of obesity. Obes Rev. 2011;12(2): 131-141.

20. Finkelstein MM. Obesity, cigarette smoking and the cost of physicians' services in Ontario. Can J Public Health. 2001;92(6):437-440.

21. Tsai AG, Williamson DF, Glick HA. Direct medical cost of overweight and obesity in the USA: a quantitative systematic review. Obes Rev. 2011;12(1):50-61.
22. Moffatt E, Shack LG, Petz GJ, Sauvé JK, Hayward K, Colman R. The cost of obesity and overweight in 2005: a case study of Alberta, Canada. Can J Public Health. 2011;102(2):144-148.

23. Shields M, Grober SC, Treblay MS. Estimates of obesity based on self-report versus direct measures. Health Rep. 2008;19(2):61-76.

24. Chiolero A, Peytremann-Bridevaux I, Paccaud F. Associations between obesity and health conditions may be overestimated if self-reported body mass index is used. Obes Rev. 2007;8(4):373-374.

25. Shields M, Connor Gorber S, Janssen I, Tremblay MS. Bias in selfreported estimates of obesity in Canadian health surveys: An update on correction equations for adults. Health Rep. 2011;22(3). Available from: http://www.statcan.gc.ca/pub/82-003-x/2011003/article/11533eng.htm. Accessed October 3, 2011.

26. Kuk JL, Ardern CI, Church TS, et al. Edmonton Obesity Staging System: association with weight history and mortality risk. Appl Physiol Nutr Metab. 2011;36(4):570-576.

27. Padwal RS, Pajewski NM, Allison DB, Sharma AM. Using the Edmonton obesity staging system to predict mortality in a populationrepresentative cohort of people with overweight and obesity. CMAJ. 2011;183(14):E1059-E1066.

28. O'Reilly D, Hopkins R, Blackhouse G, et al. Long-term cost-utility analysis of a multidisciplinary primary care diabetes management program in Ontario. Canadian Journal of Diabetes. 2007;31(3):205-214.
ClinicoEconomics and Outcomes Research

\section{Publish your work in this journal}

ClinicoEconomics \& Outcomes Research is an international, peerreviewed open-access journal focusing on Health Technology Assessment, Pharmacoeconomics and Outcomes Research in the areas of diagnosis, medical devices, and clinical, surgical and pharmacological intervention. The economic impact of health policy and health systems

\section{Dovepress}

organization also constitute important areas of coverage. The manuscript management system is completely online and includes a very quick and fair peer-review system, which is all easy to use. Visit http://www.dovepress.com/testimonials.php to read real quotes from published authors. 